\title{
Systematic Review \\ The Coronary Arteries in Adults after the Arterial Switch Operation: A Systematic Review
}

\author{
Leo J. Engele 1,2(D), Barbara J. M. Mulder ${ }^{1,2}$, Jan W. Schoones ${ }^{3,+}{ }^{\text {, Philippine Kiès }}{ }^{4}$, Anastasia D. Egorova 4 (D), \\ Hubert W. Vliegen ${ }^{4}$, Mark G. Hazekamp ${ }^{5}$, Berto J. Bouma ${ }^{1,2, \ddagger}$ and Monique R. M. Jongbloed $4,6, *, \ddagger \mathbb{D}$
}

1 Center for Congenital Heart Disease Amsterdam-Leiden (CAHAL), Department of Clinical and Experimental Cardiology, Amsterdam Cardiovascular Sciences, Heart Centre, Amsterdam UMC, University of Amsterdam, 1105 AZ Amsterdam, The Netherlands; 1.j.engele@amsterdamumc.nl (L.J.E.); b.j.mulder@amsterdamumc.nl (B.J.M.M.); b.j.bouma@amsterdamumc.nl (B.J.B.)

2 Netherlands Heart Institute, 3511 EP Utrecht, The Netherlands

3 Directorate of Research Policy, Leiden University Medical Center, 2333 ZA Leiden, The Netherlands; j.w.schoones@lumc.nl

4 Center for Congenital Heart Disease Amsterdam-Leiden (CAHAL), Department of Cardiology, Leiden University Medical Center, 2333 ZA Leiden, The Netherlands; p.kies@lumc.nl (P.K.); a.egorova@lumc.nl (A.D.E.); h.w.vliegen@lumc.nl (H.W.V.)

5 Center for Congenital Heart Disease Amsterdam-Leiden (CAHAL), Department of Cardiothoracic Surgery, Leiden University Medical Center, 2333 ZA Leiden, The Netherlands; m.g.hazekamp@lumc.nl

6 Center for Congenital Heart Disease Amsterdam-Leiden (CAHAL), Department of Anatomy and Embryology, Leiden University Medical Center, 2333 ZA Leiden, The Netherlands

* Correspondence: m.r.m.jongbloed@lumc.nl

$\dagger$ Directorate of Research Policy (Formerly: Walaus Library).

check for updates

Citation: Engele, L.J.; Mulder, B.J.M.; Schoones, J.W.; Kiès, P.; Egorova, A.D.; Vliegen, H.W.; Hazekamp, M.G.; Bouma, B.J.; Jongbloed, M.R.M. The Coronary Arteries in Adults after the Arterial Switch Operation: A Systematic Review. J. Cardiovasc. Dev. Dis. 2021, 8, 102. https://doi.org/ $10.3390 /$ jcdd 8090102

Academic Editor: Andy Wessels

Received: 6 July 2021

Accepted: 21 August 2021

Published: 26 August 2021

Publisher's Note: MDPI stays neutral with regard to jurisdictional claims in published maps and institutional affiliations.

Copyright: (c) 2021 by the authors. Licensee MDPI, Basel, Switzerland. This article is an open access article distributed under the terms and conditions of the Creative Commons Attribution (CC BY) license (https:/ / creativecommons.org/licenses/by/ $4.0 /)$. $\ddagger$ These authors contributed equally to the manuscript.

\begin{abstract}
Coronary artery status in adults long after the arterial switch operation (ASO) is unclear. We conducted a systematic review to provide an overview of coronary complications during adulthood and to evaluate the value of routine coronary imaging in adults after ASO, in light of current guidelines. Articles were screened for the inclusion of adult ASO patients and data on coronary complications and findings of coronary imaging were collected. A total of 993 adults were followed with a median available follow-up of only 2.0 years after reaching adulthood. Myocardial ischemia was suspected in $17 / 192$ patients $(8.9 \%)$. The number of coronary interventions was four $(0.4 \%)$, and coronary death was reported in four $(0.4 \%)$ patients. A lack of ischemia-related symptoms cannot be excluded because innervation studies indicated deficient cardiac innervation after ASO, although data is limited. Anatomical high-risk features found by routine coronary computed tomography (cCT) included stenosis (4\%), acute angle (40\%), kinking (24\%) and inter-arterial course (11\%). No coronary complications were reported during pregnancy $(n=45)$, although, remarkably, four $(9 \%)$ patients developed heart failure. The 2020 European Society of Cardiology (ESC) guidelines state that routine screening for coronary pathologies is questionable. Based on current findings and in line with the 2018 American ACC/AHA guidelines a baseline assessment of the coronary arteries in all ASO adults seems justifiable. Thereafter, an individualized coronary follow-up strategy is advisable at least until significant duration of follow-up is available.
\end{abstract}

Keywords: transposition of the great arteries; arterial switch operation; coronary artery; coronary complications; imaging

\section{Introduction}

Transposition of the great arteries (TGA) is a congenital heart defect with a prevalence of approximately 4.7 per 10,000 newborns and represents $5 \%$ to $7 \%$ of all congenital heart disease (CHD) [1,2]. Today, the arterial switch operation (ASO) is the operation of choice for anatomical correction in newborns with TGA and selected cases of double outlet right 
ventricle (DORV). The ASO is also part of the double switch in congenitally corrected TGA (ccTGA) patients. The ASO was performed successfully for the first time by Jatene and colleagues in 1975 [3,4], and after a transition period in the 1980s, it has now replaced the atrial switch procedures in the vast majority of cases. Translocation of the coronary arteries is the most critical step during the arterial switch procedure. The coronary arteries are excised from the aorta (neo-pulmonary trunk) after which the coronary artery is sutured into the pulmonary artery (neo-aorta) (Figure 1). Several factors contribute to a successful coronary transfer, including a sufficient length of the coronary artery and the coronary anatomy. Coronary events are an important cause of death and most often occur early after ASO; previous studies reported a prevalence of coronary obstruction after ASO from $2 \%$ to $11 \%$ in children $[5,6]$.

\section{1) Coronary artery excision and transfer}

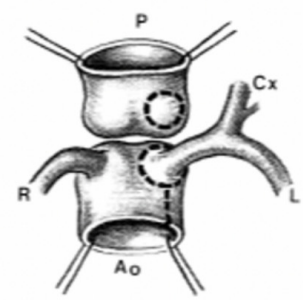

A1. Button-technique: Incision ostium LAD and $\mathrm{Cx}$

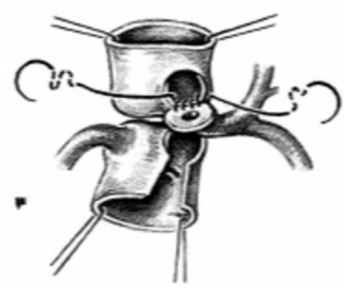

A2. Transfer LAD and Cx.

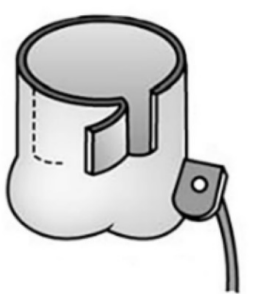

\section{B1. Trap-door technique} Incision right coronary artery

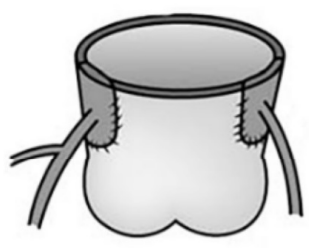

B2. Right and left coronary artery sutured in neo-aorta

\section{2) Coronary buttons}
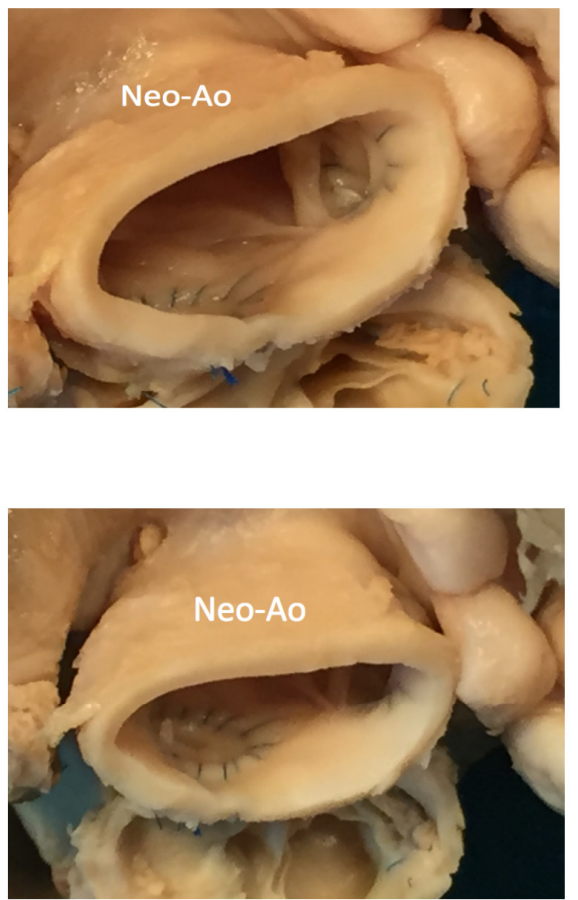

Figure 1. (1) Operation techniques for translocation of the coronary arteries during the arterial switch operation. $(\mathbf{A 1}, \mathbf{A 2})$ Button-technique of coronary artery excision and transfer to the neo-aorta in most common type (1R-2LCx). (B1,B2) Trap-door technique of coronary artery excision and transfer to the neo-aorta. (2) Coronary buttons in the neo-aorta in a patient with transposition of the great arteries after arterial switch. Abbreviations: LAD = left anterior descending artery, $\mathrm{Cx}=$ circumflex artery, Neo-Ao = Neo-aorta. Note. Images A1 and A2-1D adapted with approval of the author from the thesis: 'The Arterial Switch Operation-Rationale, Results, Perspectives, by J.M. Quaegebeur, 1986, Chapter IV, p127, ISBN 90-9001327. Images B1 and B2 are adapted from 'The importance of neo-aortic root geometry in the arterial switch operation with the trap-door technique in the subsequent development of aortic valve regurgitation', by Jhang et al. European Journal of Cardio-thoracic Surgery, 2012 Nov; 42(5): 794-92013; 96: 1390-1397. Copyright 2021 by Copyright Clearance Center.

Less is known about the long-term patency of the translocated coronary arteries and the occurrence of myocardial ischemia during adulthood. Potential mechanisms for myocardial ischemia after translocation of the coronary arteries include coronary kinking, anatomical distortion, stretching, and extrinsic compression. Furthermore, growth of the 
neo-aorta and proximal coronary arteries during lifetime may result in an increased risk for anatomical high-risk features of the coronary arteries including acute angle, mechanical kinking or stretching [7-9]. Patients with an unusual anatomical coronary pattern may be at higher risk for the occurrence of late coronary complications [10]. Due to cardiac denervation after dissecting the cardiac plexus during ASO, patients with a coronary artery stenosis may be asymptomatic. Previous long-term follow-up studies in children reported significant coronary stenosis after ASO in asymptomatic patients [6,7].

Due to the lifelong risk of coronary complications after the ASO, periodic surveillance of the coronary arteries is recommended by current guidelines [11,12]. Non-invasive examinations for detecting myocardial ischemia with ECG, stress echocardiography, exercise testing, and perfusion scintigraphy have shown low sensitivity for detecting significant coronary stenosis [6]. However, Cardiovascular Magnetic Resonance (CMR) and coronary computed tomography ( $\mathrm{cCT}$ ) can be of value in timely detection of coronary complications. There is currently no consensus regarding the optimal follow-up interval and imaging modality for surveillance of the translocated coronary arteries.

Currently, the group of young adult ASO patients is growing. Although many studies reported coronary complications during childhood, a structured overview of available literature concerning coronary status in adults is lacking. Therefore, we performed a systematic review to provide an overview of currently available literature reporting on the incidence of coronary complications specifically during adulthood and the value of coronary imaging in adults after ASO. Besides, the effects of pregnancy on coronary outcomes and studies on cardiac innervation, given the potential relevance for symptomatology, were analysed. Findings will be discussed in light of current coronary follow-up recommendations after the ASO.

\section{Materials and Methods}

This systematic review was conducted according to the Preferred Reporting Items for Systematic Reviews and Meta Analyses (PRISMA) checklist [11]. Quality assessment of included long-term follow-up cohort studies was performed with the Newcastle Ottawa Scale (NOS) [13]. Studies were scored on the following two domains: (1) selection, including representativeness of the exposed cohort, ascertainment of exposure, and demonstration that the outcome of interest was not present at the start of the study; (2) outcome, including assessment of outcome, follow-up period, and adequacy of follow-up. The maximum number of awarded stars was six.

\subsection{Search Strategy}

A comprehensive literature search was performed in PubMed, Embase, Web of Science, Cochrane Library and Emcare. Language was restricted to English and German. Because we focused on the outcomes in adults, only articles which were published between 1994 (18 years after introduction of the ASO) and August 2020 were selected. The search strategy was carried out by using key words for arterial switch, transposition of the great arteries, coronary outcome, long-term outcome, and ischemia. (For complete query: see Appendix A). Duplicate articles were removed.

\subsection{Selection Criteria}

Cohort studies with TGA, DORV or ccTGA patients after ASO were included. Longterm outcome on coronary interventions or coronary death in adults ( $\geq 18$ years) was retrieved. Because the purpose was to evaluate the long-term coronary status after arterial switch, patients with one-stage repair, two-stage repair, and late arterial switch were all included in analysis. Furthermore, cross-sectional coronary imaging studies investigating coronary anatomy or myocardial perfusion with any imaging modality were considered eligible for the review. Finally, studies reporting cardiac outcomes during pregnancy, cardiac innervation, and case reports on coronary complications were included. Studies reporting other than coronary outcome in adults after ASO, description of outcome exclusively in 
children $(<18$ year), or patients with a non-identifiable age were excluded. In addition, reviews, editorials and articles in which the full-text could not be retrieved were excluded. Reference lists of reviews were searched for eligible articles which were not identified in the literature search.

\subsection{Definitions}

The literature search was restricted to adults (18 years or older) because the purpose was to evaluate the long-term patency of the coronary arteries without focusing on the early and midterm outcome. Coronary complications were defined as percutaneous coronary intervention, coronary surgery or coronary death. The following causes of death were interpreted as coronary related: (aborted) sudden cardiac death or death related to proven or possible ischemic heart failure. All imaging techniques performed in adults to assess coronary anatomy or coronary function were considered eligible for systematic review. When duplication of patient data in studies from the same institutions were suspected, the study with the highest number of ASO adults with description of coronary complications was included.

\subsection{Data Extraction and Appraisal}

All abstracts were screened for eligibility by two independent observers (LJE and MRMJ). In case of disagreement, differences between the observers' judgements were discussed to seek consensus. In each of the included long-term follow-up papers, data were collected on the number of included adults, the number of coronary complications, and the follow-up time, using data derived from full-text, tables, and overall Kaplan-Meier curves. One observer (LJE) performed a quality assessment of the included papers using the NOS. In patients with coronary complications, data were collected on NYHA class and presence of chest pain when available. Data on the number of adults and duration of follow-up collected from Kaplan-Meier curves were categorized into the following age groups: 18-20 years, 20-25 years, 25-30 years, older than 30 years. It was not possible to identify the censored age for each patient from the Kaplan-Meier curves. Therefore, we were not able to include patients who were censored at the age of 18 and 19 years. Mean follow-up was calculated with the follow-up time derived from Kaplan-Meier curves.

In cross-sectional anatomical and physiological imaging studies, data on the imaging technique, the number of patients with anatomical high-risk features, perfusion defects, ischemia, and coronary intervention were retrieved. In addition, data of patients with suspicion of myocardial ischemia were collected. Anatomical high-risk features of the proximal coronary arteries following ASO were defined as: acute angle take-off ( $\leq 30$ degree), interarterial course, ostial stenosis, and/or kinking. These coronary features have been associated with myocardial ischemia in CHD cohorts [14,15].

Suspicion of myocardial ischemia was based on symptoms or ST-segment deviation during exercise tests. Inclusion of imaging studies was based on the median or mean age ( $\geq 18$ years) of the ASO cohort; therefore, the potential presence of individual patients in this cohort with an age under 18 years could not be excluded. The following data were extracted from case reports: type of examination, type of intervention, presence of chest pain. From pregnancy studies, data on the number of coronary complications, ventricular rhythm disorders, and left ventricular function were collected. The technique and outcome were retrieved from cardiac innervation studies.

\section{Results}

\subsection{Study Selection}

A total of 893 studies were identified (Figure 2). The number of excluded studies by abstract screening was 658; most of these reported outcome exclusively in children ( $<18$ years) or focused on atrial switch procedures. The remaining 235 articles were assessed for eligibility by full-text screening. After full-text assessment, 46 studies were included with the following study designs: 27 long-term follow-up cohort studies, 3 cross-sectional 
imaging studies, and 4 case reports. In addition, studies focusing on the following aspects were included: late arterial switch studies $(n=4)$, double switch studies $(n=2)$, pregnancy studies $(n=4)$, and sympathetic innervation studies $(n=2)$.

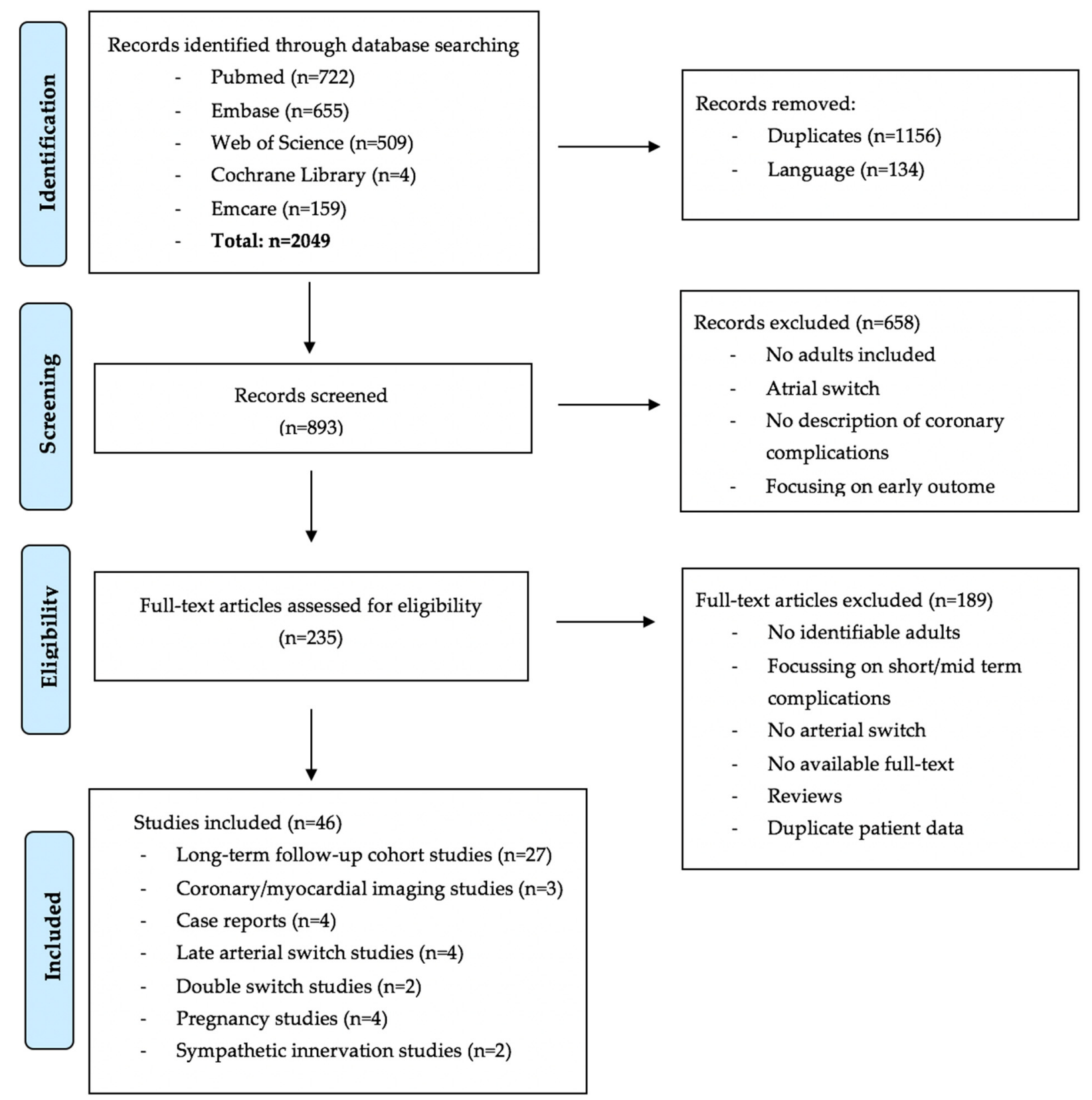

Figure 2. Prisma flow diagram.

\subsection{Long-Term Follow-Up Cohort Studies}

A total number of 993 ASO patients were followed for at least 18 years in the long-term follow-up cohort studies [16-41] (Table 1). Figure 3A shows the freedom from coronary complications; the number at risk at 18 years is 993 patients. However, at the age of 25 years the number at risk is only 130 patients, which is explained by the relatively young age of the adult patients described in the included papers. The median follow-up duration into adulthood was 2.0 years (mean 2.8 years). Of the 993 adult ASO patients that were included, a total of $8(0.8 \%)$ coronary-related events were reported. Specifically, four patients $(0.4 \%)$ underwent a coronary intervention (percutaneous coronary intervention (PCI) or coronary artery bypass grafting $(C A B G))$ for coronary stenosis $(n=2)$ or occlusion $(n=2)$. One 
patient $(0.1 \%)$ who underwent coronary intervention was asymptomatic. Coronary death was reported in four patients $(0.4 \%)$ : three patients died due to sudden cardiac death, and one patient was found in asystole. All coronary complications are summarized in Table 2. Seventeen out of $192(8.9 \%)$ patients $[19,27,42]$ were suspected to have myocardial ischemia based on symptoms or exercise testing; after additional examinations, 3 out of 17 patients underwent coronary intervention (PCI or CABG).

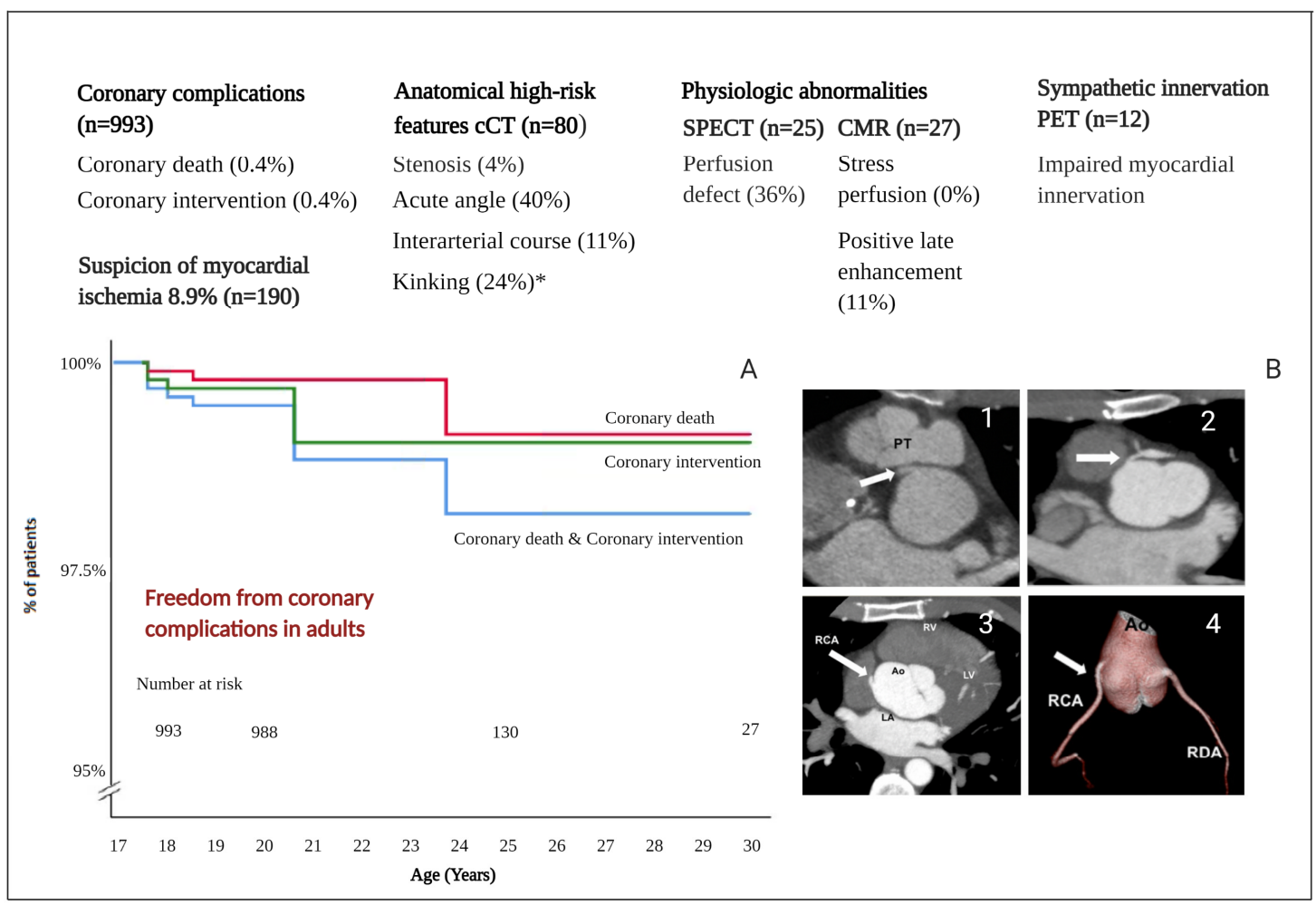

Figure 3. Coronary artery status in adults after ASO. Legend: (A). Freedom from coronary complications in adults after ASO. (B). Anatomical high-risk features on coronary computed tomography. (B1) Interarterial course of the right coronary artery. (B2) A significant proximal left anterior descending coronary artery stenosis. (B3) Acute angle right coronary artery (axial slice). (B4) Acute angle right coronary artery (three-dimensional reconstruction). Abbreviations: $\mathrm{cCT}=\mathrm{coronary}$ computer tomography, SPECT = single-photon emission computer tomography, CMR = cardiovascular magnetic resonance, PET = position emission tomography, RDA = Ramus Descendens anterior, RCA = Right Coronary Artery. * only 1 study did describe the presence of kinking $(n=50)$. Note. CT images B1 and B2 are adapted from 'Variation in Coronary Anatomy in Adult Patients Late After Arterial Switch Operation: A Computed Tomography Coronary Angiography Study', by Veltman et al. Ann Thorac Surg, 2013; 96: 1390-1397. Copyright 2021 by Copyright Clearance Center.

\subsection{Coronary Imaging Studies}

Two studies $[9,42]$ examined coronary anatomy in adults using routine cCT. The combined results of both studies demonstrated that 4 out of $80(5 \%)$ patients were suspected of coronary stenosis. Three of these patients had no anginal complaints and did not have ischemia at functional testing with single-photon emission computed tomography (SPECT); therefore, no further investigations were performed. In the fourth patient, an angiogram was performed which showed significant proximal luminal narrowing caused by a fibrotic lesion, after which a percutaneous intervention with stent implantation was performed. Other anatomical high-risk features identified by cCT included the following: presence of an acute angle $(40 \%)$, interarterial course $(11 \%)$ and proximal kinking $(24 \%)$. Additional stress SPECT did not show a correlation between perfusion defects and the presence of these high-risk features. Another imaging study in a cohort of 27 ASO adults compared cardiovascular magnetic resonance (CMR) and SPECT under identical physiological circumstances [43]. Nine out of 25 patients (36\%) were reported as having perfusion defects 
on SPECT; however, CMR stress perfusion with dipyridamole was visually normal in all these patients. A summary of coronary abnormalities of all imaging studies $(n=3)$ is provided in Table 3 and Figure 3.

Table 1. Overview of the extracted studies on coronary complications across different age groups in ASO adults.

\begin{tabular}{|c|c|c|c|c|c|c|c|}
\hline \multirow[b]{3}{*}{ Author } & \multirow[b]{3}{*}{ Patients $\geq 18$ Year } & \multicolumn{6}{|c|}{ Age Groups (Years) } \\
\hline & & & $18-20$ & $20-25$ & $25-30$ & $>30$ & \\
\hline & & $\begin{array}{l}\text { Maximum } \\
\text { Follow-Up in } \\
\text { Adulthood (Yr) }\end{array}$ & $\begin{array}{c}\text { Event } / \text { n. at } \\
\text { Risk }\end{array}$ & $\begin{array}{c}\text { Event/n. at } \\
\text { Risk }\end{array}$ & $\begin{array}{c}\text { Event } / \mathrm{n} \text {. at } \\
\text { Risk }\end{array}$ & $\begin{array}{c}\text { Event } / \mathrm{n} \text {. at } \\
\text { Risk }\end{array}$ & $\begin{array}{l}\text { Newcastle } \\
\text { Ottawa Scale }\end{array}$ \\
\hline Fricke et al. [16] & 183 & 2 & $0 / 183$ & - & - & - & 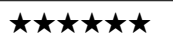 \\
\hline Khairy et al. [17] & 148 & 7 & $0 / 148$ & $1 / 28$ & - & - & $\hat{\star} \hat{\star} \star \hat{\star} \star \hat{\star}$ \\
\hline Moll et al. [18] & 136 & 7 & $0 / 136$ & $0 / 4$ & - & - & 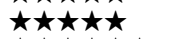 \\
\hline Kempny et al. [19] & 112 & 20 & $3 / 112$ & $1 / 67$ & $0 / 20$ & $1 / 1$ & 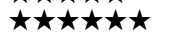 \\
\hline Tobler et al. [20] & 60 & 7 & $1 / 60$ & $0 / 10$ & - & - & 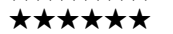 \\
\hline Santens et al. [21] & 52 & 12 & $0 / 52$ & $0 / 4$ & $0 / 4$ & - & 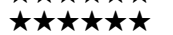 \\
\hline Baruteau et al. [22] & 47 & 7 & $0 / 47$ & $0 / 6$ & - & - & 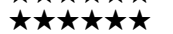 \\
\hline Lo Rito et al. [23]. & 44 & 2 & $0 / 44$ & - & - & - & 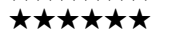 \\
\hline Vida et al. [24] & 44 & 12 & $0 / 44$ & $0 / 1$ & $0 / 1$ & - & 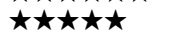 \\
\hline Oda et al. [25] & 40 & 2 & $0 / 40$ & - & - & - & 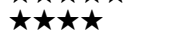 \\
\hline Raissadati et al. [26] & 34 & 12 & $0 / 34$ & $0 / 1$ & $0 / 1$ & - & 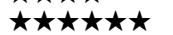 \\
\hline Ruys et al. [27] & 21 & 2 & $0 / 21$ & - & - & - & $\hat{\star} \hat{\star} \star \hat{\star} \star \hat{\star} \hat{\star}$ \\
\hline Hörer et al. [19] & 17 & 2 & $0 / 17$ & - & - & - & $\hat{\star} \hat{\star} \star \hat{\star} \star \hat{\star}$ \\
\hline Shivaram et al. [29] & 13 & 12 & $0 / 13$ & $0 / 3$ & $0 / 1$ & - & 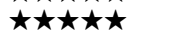 \\
\hline Lalezari et al. [44] & 11 & 7 & $0 / 11$ & $0 / 2$ & - & - & 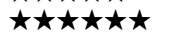 \\
\hline Lim et al. [30] & 9 & 2 & $0 / 9$ & - & - & - & $\star \star \star \star \star \star$ \\
\hline Choi et al. [31] & 5 & $\overline{7}$ & $0 / 5$ & $0 / 3$ & - & - & 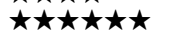 \\
\hline Rudra et al. [32] & 4 & 2 & $0 / 4$ & - & - & - & 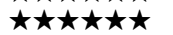 \\
\hline Gerelli et al. [33] & 2 & 2 & $0 / 2$ & - & - & - & 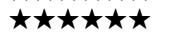 \\
\hline De Praetere et al. [34] & 3 & 7 & $0 / 3$ & $0 / 1$ & - & - & 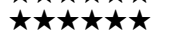 \\
\hline Hayes et al. [45] & 2 & 2 & $0 / 2$ & - & - & - & 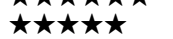 \\
\hline Hutter et al. [36] & 1 & 2 & $0 / 1$ & - & - & - & 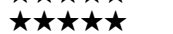 \\
\hline Manso et al. [37] & 1 & 2 & $0 / 1$ & - & - & - & $\hat{\star \star \star \star \star \star \star \star \star ~}$ \\
\hline El-Segaier et al. [38] & 1 & 0 & $1 / 1$ & - & - & - & $\hat{\star} \star \hat{\star} \star \hat{\star} \star \hat{\star} \star \hat{~}$ \\
\hline Gorler et al. [39] & 1 & 2 & $0 / 1$ & - & - & - & 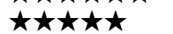 \\
\hline Arnaz et al. [40] & 1 & 2 & $0 / 1$ & - & - & - & $\star \star \star \star \star$ \\
\hline Shim et al. [41] & 1 & 2 & $0 / 1$ & - & - & - & 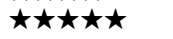 \\
\hline Total & 993 & median 2.0 & $5 / 993$ & $2 / 130$ & $0 / 27$ & $1 / 1$ & \\
\hline
\end{tabular}

Table 2. Clinical data of ASO patients with coronary complication during adulthood.

\begin{tabular}{llllll}
\hline Author & Age at ASO & Age at Event (y) & Coronary Anatomy & Chest Pain & NYHA Class \\
\hline Kempny et al. [19] & - & 18 & - & - & - \\
Kempny et al. [19] & - & 18 & - & - & - \\
Tobler et al. [20] & 6 days & 18 & Usual & - & PCI/CABG \\
El-Sagaier et al. [38] & 4 days & 18 & Usual & No & - \\
Kempny et al. [19] & 7 months & 19 & Cx from RCA & - & PCI/CABG \\
Kempny et al. [19] & - & 21 & Single RCA & - & III \\
Khairy et al. [17] & - & 24 & Usual & - & PCI LCA \\
Kempny et al. [19] & 10 y & 38 & Sudden cardiac death & PCI/CABG \\
\hline
\end{tabular}

Abbreviations: NYHA = New York Heart Association, $\mathrm{CABG}=$ coronary artery bypass graft, $\mathrm{ASO}=$ arterial switch operation, $\mathrm{PCI}=$ percutaneous coronary intervention, $\mathrm{y}=$ years, $\mathrm{LCA}=$ left coronary artery $\mathrm{RCA}=$ right coronary. artery, $\mathrm{Cx}=$ circumflex.

Table 3. Description of findings in cardiac imaging studies in adults after ASO.

\begin{tabular}{|c|c|c|c|c|c|c|c|c|c|c|c|c|}
\hline & & & & & & cCT & & SPECT & & CMR & Angiogran & \\
\hline Author & $\mathbf{N}$ & Age & $\begin{array}{l}\text { Common } \\
\text { Coronary } \\
\text { Anatomy n } \\
(\%)\end{array}$ & $\begin{array}{l}\text { Coronary } \\
\text { Stenosis } \\
\text { n }(\%)\end{array}$ & $\begin{array}{l}\text { Inter- } \\
\text { Arterialn } \\
(\%)\end{array}$ & $\begin{array}{l}\text { Acute } \\
\text { Anglen } \\
(\%)\end{array}$ & $\begin{array}{l}\text { Kinkingn } \\
(\%)\end{array}$ & $\begin{array}{l}\text { Perfusion } \\
\text { Defectn } \\
\text { (\%) }\end{array}$ & $\begin{array}{l}\text { Ischemia } \\
\text { n (\%) }\end{array}$ & $\begin{array}{l}\text { Ischemia } \\
\text { n/Total n }\end{array}$ & $\begin{array}{l}\text { Coronary } \\
\text { Stenosis } \\
\text { n/Total n }\end{array}$ & $\begin{array}{l}\text { Coronary } \\
\text { Interven- } \\
\text { tion }(\%)\end{array}$ \\
\hline Michalak [40] & 50 & 23 & - & $3(6 \%)$ & $4(8 \%)$ & $25(50 \%)$ & $12(24 \%)$ & $3(6 \%)$ & $0(0 \%)$ & - & - & $0(0 \%)$ \\
\hline Veltman [8] & 30 & 22.0 & $24(80)$ & $1(3 \%)$ & $5(17 \%)$ & $7(23 \%)$ & - & $3(14 \%)$ & $0(0 \%)$ & - & $1 / 1$ & $1(3.3 \%)$ \\
\hline Tobler [39] & 27 & 21 & $18(67)$ & - & - & - & - & $13(52 \%)$ & $8(32 \%)$ & $0 / 27$ & $0 / 1$ & $0(0 \%)$ \\
\hline
\end{tabular}

Abbreviations: $\mathrm{CAD}=$ coronary artery disease, $\mathrm{n}=$ number of patients, total $\mathrm{n}=$ total number of examined patients, $\mathrm{CT}=$ computer tomography, SPECT = single photon emission computed tomography, CMR = cardiovascular magnetic resonance.

\subsection{Case Reports}

The literature search identified four case reports [46-49] (Table 4) in which a successful coronary intervention was reported in adults with a significant coronary lesion. Two patients were diagnosed with a significant stenosis of the left main stem. One patient 
presented an acute coronary syndrome due to compression of the left main stem. The final patient had subtotal RCA occlusion after pulmonary artery surgery. The presence of chest pain was described in three patients; one patient was asymptomatic.

Table 4. Case reports reporting coronary intervention in ASO adults.

\begin{tabular}{llllll}
\hline Author & Age & Reason for Intervention & Examination & Chest Pain & Intervention \\
\hline Quarrie et al. [45] & $18 \mathrm{yr}$ & $\begin{array}{l}\text { Left main stenosis } \\
\text { ACS due to left main stem } \\
\text { compression }\end{array}$ & SPECT Angio & No & Surgery LIMA-LAD \\
Stoll et al. [46] & $27 \mathrm{yr}$ & $\begin{array}{l}\text { subtotal occlusion RCA after } \\
\text { patch plasty for PA stenosis }\end{array}$ & Angiography & Yes & rurgery: defect was \\
reroofed
\end{tabular}

Abbreviations: ACS = acute coronary syndrome, SPECT $=$ single photon emission computed tomography, $\mathrm{CMR}=$ cardiovascular magnetic resonance, $\mathrm{CT}=$ computer tomography, $\mathrm{y}=$ years, LIMA = left internal mammary artery, LAD = left artery descending, $\mathrm{PA}=$ pulmonary artery.

\subsection{Pregnancy Studies}

Four studies described the outcome in patients during pregnancy [50-53] (Table 5). 45 patients were followed peri- and post-partum during 73 pregnancies. During these pregnancies, no coronary events were reported. However, two patients $(4.4 \%)$ had decrease in $\mathrm{LV}$ function, and in four patients $(9 \%)$, heart failure requiring diuretics treatment was reported. Nonsustained ventricular tachycardia was described in four $(9 \%)$ patients. These outcomes were not associated with coronary events but could partly be attributed to valvular heart disease. Risk factor for adverse outcome included older age at ASO and higher maternal age [50-53].

Table 5. Cardiovascular outcome peri- and post-partum in ASO adults.

\begin{tabular}{|c|c|c|c|c|c|c|c|c|c|}
\hline \multirow[b]{2}{*}{ Author } & \multirow[b]{2}{*}{ All pt } & \multirow[b]{2}{*}{$\begin{array}{l}\text { Number of } \\
\text { Pregnancies }\end{array}$} & \multirow[b]{2}{*}{ Age } & \multicolumn{3}{|c|}{ Peripartum } & \multicolumn{3}{|c|}{ Postpartum } \\
\hline & & & & $\begin{array}{c}\text { Coronary } \\
\text { Event, } n\end{array}$ & $\begin{array}{c}\text { Heart } \\
\text { Failure, } \mathrm{n}\end{array}$ & nsVT, n & nsVT, n & $\begin{array}{l}\text { Decrease LV } \\
\text { Function, } n\end{array}$ & $\begin{array}{c}\text { Heart } \\
\text { Failure, } n\end{array}$ \\
\hline Stoll, V.M., et al. [49] & 15 & 25 & 23 & 0 & & & & & \\
\hline Tobler, D., et al. [50] & 9 & 17 & 22 & 0 & & & 1 & 2 & \\
\hline Fricke, T.A., et al. [51] & 11 & 17 & 29 & 0 & 1 & & & & \\
\hline Horiuchi, C., et al. [52] & 10 & 14 & 29 & 0 & 0 & 2 & 3 & 0 & 3 \\
\hline
\end{tabular}

Abbreviations: $\mathrm{pt}=$ patient, $\mathrm{nsVT}=$ non sustained ventricular tachycardia, $\mathrm{n}=$ number of patients, $\mathrm{LV}=$ left ventricular.

\subsection{Late Arterial Switch and Double Switch}

In 12 patients, a late arterial switch operation was performed during adulthood [54-57] (Table 6). Eleven patients were free from coronary complications during follow-up (range: three months to five years). One patient died five days post-operatively because of low cardiac output syndrome. The outcome of double switch was reported in two adult patients with ccTGA $[58,59]$ (Table 7). Both patients, operated at the age of 14 years and within the first year of life, were free from coronary complications at 5- and 20-year follow-up, respectively.

Table 6. Coronary complications during adulthood in patients after late arterial switch.

\begin{tabular}{lcccc}
\hline Author & $\begin{array}{c}\text { n. of Adult } \\
\text { Patients }\end{array}$ & Age at ASO & Follow-Up & Coronary Complications \\
\hline Cetta et al. [53] & 1 & $36 \mathrm{yr}$ & $3 \mathrm{~m}$ & no \\
Padalino et al. [55] & 1 & $23 \mathrm{yr}$ & $1 \mathrm{yr}$ & no \\
Watanabe et al. [56] & 8 & $>20 \mathrm{yr}$ & $5 \mathrm{yr}$ & no \\
Maeda et al. [54] & 2 & $18.6 \mathrm{yr} \& 32.4 \mathrm{yr}$ & - & 1 patient died 5 days after ASO due to \\
low cardiac ouput syndrome
\end{tabular}


Table 7. Coronary complications in adults after double switch.

\begin{tabular}{lcccc}
\hline Author & n. of Adult Patients & Age at ASO & Follow-Up & Coronary Complications \\
\hline Uno et al. [57] & 1 & $14 \mathrm{yr}$ & $5 \mathrm{yr}$ & no \\
Konstantinov et al. [58] & 1 & within the first year & $20 \mathrm{yr}$ & no \\
\hline
\end{tabular}

Abbreviations: $\mathrm{n}$ = number, $\mathrm{ASO}=$ arterial switch operation, $\mathrm{yr}=$ year.

\subsection{Cardiac Sympathetic Innervation}

Two studies $[60,61]$ were found on cardiac sympathetic innervation exclusively in adults. Possner and colleagues [60] demonstrated that cardiac sympathetic innervation, measured by $\left[{ }^{11} \mathrm{C}\right]$ meta-hydroxyephedrine uptake, was significantly lower in $12 \mathrm{ASO}$ patients compared to 10 healthy individuals, indicating impaired myocardial innervation long-term after ASO. Furthermore, there was no difference in myocardial blood flow (MBF) response after the cold pressor test; however, when corrected for heart rate, the MBF response was lower in ASO patients. This may be explained by an increased release of catecholamines compensating the deficient innervation. The second study [61] investigated myocardial sympathetic innervation in nine ASO patients and nine Rastelli patients by measuring ${ }^{11} \mathrm{C}$ epinephrine (EPI) retention with positron-emission tomography (PET). In eight ASO patients signs of reinnervation were found, one patient was suspected to have complete denervation (EPI-retention $<7 \% / \mathrm{min}$ ). An association was found between reduced EPI retention and patients undergoing more than one cardiothoracic operation. A summary of findings is provided in Table 8.

Table 8. Description of findings in cardiac innervation studies in adults after ASO.

\begin{tabular}{|c|c|c|c|c|c|c|c|c|c|}
\hline \multirow[b]{3}{*}{ Author } & \multirow[b]{3}{*}{ All pt } & \multirow[b]{3}{*}{ Age } & \multirow[b]{3}{*}{ Examination } & \multirow{3}{*}{$\begin{array}{c}\text { cCT } \\
\text { (Ostial) } \\
\text { Stenosis }\end{array}$} & \multicolumn{3}{|c|}{ H2O PET } & \multicolumn{2}{|l|}{ PET } \\
\hline & & & & & \multicolumn{3}{|c|}{ Myocardial Blood Flow (mL/min/g) } & \multicolumn{2}{|c|}{ EPI Retention } \\
\hline & & & & & Rest & Adenosine & Cold Pressor Test & Mean $(\% / \mathrm{min})$ & $<7 \% /$ min \\
\hline Possner et al. [60] & 12 & 22.5 & PET \& cTA & 0 & $0.66 \pm 0.08$ & $2.23 \pm 1.19$ & $0.99 \pm 0.20$ & - & - \\
\hline Kuehn et al. [61] & 9 & 20.8 & PET & - & - & - & - & $12.83 \pm 1.42$ & $\mathrm{n}=1$ \\
\hline
\end{tabular}

Abbreviations: $\mathrm{cCT}=$ coronary computed tomography, $\mathrm{PET}=$ positron-emission tomography, $\mathrm{pt}=$ patient, $\mathrm{EPI}=$ epinephrine.

\section{Discussion}

This systematic review shows that the number of reported coronary complications in $993 \mathrm{ASO}$ adults is low $(0.8 \%)$, but the follow-up period after reaching the age of 18 years is limited with a median of only 2.0 years. In our opinion, the limited follow-up duration demonstrates the relevance of this systematic review to provide an overview of current available literature on coronary arterial status of the young adult ASO population because the evidence for current guideline recommendations for coronary follow-up remains limited. Compared to most previous ASO studies in children, the unique aspect of the current systematic review is that it is focused on the coronary status solely in the adult ASO population with a description of late coronary complications, including an overview of both anatomical and physiological coronary imaging studies.

\subsection{Coronary Imaging Strategy}

The review focused on the adult population of each cohort; however, similarly low numbers of coronary interventions in children are reported in two included long-term follow-up cohort studies with a routine coronary imaging strategy and a strategy where coronary imaging was performed on clinical indication. Baruteau and colleagues [22] reported the outcome of a routine coronary imaging strategy with catheterization or cCT in 200 ASO patients; this examination was performed every five years, starting from their fifth year. During a median follow-up of 17 years, coronary stenosis or occlusion was found in 4 patients ( $2 \%)$ at a median age of 9.7 years. Khairy et al. [17] described the coronary outcome in 400 ASO patients during a median follow-up of 18.7 years and 
reported myocardial infarction in 4 children (1\%). In these ASO patients, coronary imaging was performed not on a routine basis but only when clinically indicated.

\subsection{Imaging Techniques}

SPECT can be performed to detect myocardial perfusion defects in patients after ASO. In comparison with the specificity, the sensitivity for detecting coronary stenosis is low [6]. Clinical significance of perfusion abnormalities is unclear and might be related to the operation itself rather than reflecting coronary flow determined by myocardial perfusion [62]. Despite radiation exposure, $\mathrm{CCT}$ is a good non-invasive imaging technique alternative for detecting ostial and proximal coronary stenosis compared to gold standard coronary angiography [63]. In the current review, two anatomical imaging studies in adults $(\mathrm{n}=80)$ after ASO demonstrated that anatomical high-risk features, including acute angle, inter-arterial course and kinking were frequently observed at routine cCT. However, additional physiological examination by SPECT at rest and during exercise demonstrated that most of these high-risk features were not associated with significant perfusion defects. A recent systematic review from Morfaw et al. [64] reported a prevalence of any coronary anomaly of $23.0 \%$ in ASO patients older than 20 years, although it was not specified how these coronary anomalies were defined. In this review, we focused specifically on the occurrence of coronary complications and coronary high-risk features. The presence of high-risk features may be related to the technical approach of the coronary transfer and not necessarily the original coronary anatomy. This is consistent with Michalak et al. [42] who did not find an association between coronary anomaly and the presence of high-risk features. In their analysis, a proximal coronary stenosis was significantly correlated with a history of complex coronary transfer. Although, the presence of an acute angle was not associated with surgical technique.

Contradictory literature results about the association between coronary features and mortality exists. A meta-analysis by Pasquali and colleagues reported that in almost 2000 ASO patients, all operated before 1999, both an intramural course and single coronary artery anatomy were associated with a higher all-cause mortality rate. However, in a recent study [16], these abnormalities were not found to be a risk factor for death, which may be explained by the improved surgical experience with coronary high-risk features. Data regarding the impact of anatomical high-risk features on myocardial flow is limited, and further investigation is needed [65].

Existing literature on the anomalous aortic origin of a coronary artery (AAOCA) demonstrated that the presence of a high orifice, ostial stenosis, intramural course and length, inter-arterial course, acute angulation and a slit like orifice have been associated with myocardial ischemia $[14,15]$. The current 2020 ESC guideline for coronary anomalies recommends physical-stress-induced ischemia testing to examine the presence of myocardial ischemia [12]. The clinical significance of the anatomical high-risk features in the ASO population is still undetermined. Progressive neo-aortic root dilatation has been reported in adults after ASO [66]. Due to dilatation of the neo-aortic root in both radial and longitudinal directions, the angle between the coronary artery and aortic wall could decrease progressively during adulthood and may result in coronary stenosis during adult life. Veltman and colleagues [9] reported an association between larger neo-aortic root dimensions and higher coronary take-off in patients with an acute angle, suggesting that individual patient root geometry might play an additional role.

\subsection{Follow-Up Strategy and Current Findings in Relation to Guidelines Recommendations}

To determine the long-term patency of the coronary arteries in this population of young adults, a lifelong follow-up is required. Currently, the 2018 ACC and AHA guidelines for the management of adults with congenital heart disease [11] recommend at least single baseline investigation of the coronary arteries by catheter angiography or $\mathrm{CCT}$. Thereafter, the decision is made on clinical indication. The 2020 ESC guidelines on ACHD [12] recommend $\mathrm{CCT}$ when a stenosis is suspected, and they state that a routine non-invasive 
imaging of coronary arteries with $\mathrm{CCT}$ is questionable. Although the current systematic review shows a low number of reported coronary complications, it also exposes that the amount of long-term follow-up data concerning coronary status in adults is still limited. The impact of suture induced fibrosis and atherosclerotic disease on the translocated coronary arties is unclear, and patients with coronary stenosis may be less symptomatic due to decreased innervation post-cardiac surgery. Based on the number of anatomical high-risk features detected by coronary imaging, it seems justified that ASO patients should have at least a baseline assessment of the coronary anatomy with non-invasive $\mathrm{CT}$ angiography during young adulthood with additional physiological imaging in patients with high-risk anatomical features or clinical signs of myocardial ischemia.

\subsection{Limitations}

The number of adults extracted from the Kaplan-Meier curves is underestimated because most studies reported the number of patients at risk at 20 years. However, the number of patients censored at the age of 18 and 19 were not reported and therefore could not be included in this systematic review. One explanation for the low number of coronary complications reported during adulthood might be that coronary complications may occur prior to adulthood. In our analysis, these patients were not included because the event occurred during childhood, and these patients were censored before the age of 18 in Kaplan-Meier figures. Secondly, all reported data regarding anatomical high-risk features of the coronary arteries were analysed; however, not all studies described the same coronary features. Thirdly, strategies for coronary follow-up were different between studies. As a consequence of this heterogeneity, coronary interventions may have been performed more frequently in centers with a routine coronary imaging protocol. Finally, all patients after ASO including TGA, DORV and late arterial switch were included in our review because it was not possible to identify individual patients for subgroup analyses.

\section{Conclusions}

The number of reported coronary complications in 993 ASO adults during a median follow-up of 2 years is low $(0.8 \%)$. Anatomical high-risk features were frequently found by routine coronary imaging with $\mathrm{cCT}$ in ASO adults; however, the number of interventions in these patients is low, and the clinical significance of these high-risk features at longer term follow up remains unclear. Based on current findings and in line with the 2018 ACC and AHA guidelines on ACHD, we suggest a baseline assessment of the coronary arteries. Thereafter, an individualized coronary follow-up strategy seems appropriate in young adult patients after the ASO.

Author Contributions: Conceptualization: L.J.E., B.J.M.M., J.W.S., M.R.M.J.; methodology, J.W.S., L.J.E., M.R.M.J.; formal analysis, L.J.E.; resources, J.W.S., L.J.E.; writing —original draft preparation, L.J.E., B.J.M.M., J.W.S., M.R.M.J., B.J.B.; writing—review and editing, L.J.E., B.J.M.M., J.W.S., P.K., A.D.E., H.W.V., M.G.H., B.J.B., M.R.M.J.; visualization, L.J.E., M.R.M.J.; supervision, B.J.M.M., B.J.B., M.R.M.J.; funding acquisition, B.J.M.M., B.J.B., M.R.M.J. All authors have read and agreed to the published version of the manuscript.

Funding: This research was funded by the Netherlands Heart Institute (NLHI-308), grant Outreach, grant number CVON2019-002.

Acknowledgments: In respectful memory of Adriana Gittenberger-de Groot, who passionately studied coronary arterial anatomy in congenital heart disease during her life and laid the foundation of much of our knowledge on this subject.

Conflicts of Interest: The authors declare no conflict of interest. 


\section{Appendix A}

\section{Literature Search}

Pubmed: ((("Arterial Switch Operation"[Mesh] OR “Arterial Switch Operation" [tw] OR "Arterial Switch Operations" [tw] OR "arterial switch procedure" [tw] OR "arterial switch procedures" $[\mathrm{tw}] \mathrm{OR}$ "arterial switch repair" $[\mathrm{tw}] \mathrm{OR}$ "arterial switch surgery" $[\mathrm{tw}] \mathrm{OR}$ "arterial switch" $[\mathrm{tw}]$ OR "Arterial Switch Technique" $[\mathrm{tw}]$ OR "Double Switch Operation" [tw] OR "Double Switch Operations" [tw] OR "Double Switch Procedure" [tw] OR "Double Switch Procedures" $[\mathrm{tw}]$ OR "Double Switch Technique" $[\mathrm{tw}]$ OR "Double Switch" $[\mathrm{tw}]$ OR "Jatene Operation" $[\mathrm{tw}]$ OR "Jatene Procedure"[tw] OR "Jatene Technique"[tw]) AND ("coronary complications" [tw] OR "coronary complication" [tw] OR "Coronary Disease"[mesh] OR "Coronary Aneurysm" $[\mathrm{tw}]$ OR "Coronary Aneurysms" [tw] OR "Coronary Artery Disease" [tw] OR "Coronary Artery Diseases" $[\mathrm{tw}]$ OR “Coronary Disease" $[\mathrm{tw}]$ OR “Coronary Diseases" $[\mathrm{tw}]$ OR "Coronary Occlusion" [tw] OR "Coronary Occlusions" [tw] OR "Coronary Restenosis" [tw] OR “Coronary Restenoses" [tw] OR “Coronary Stenosis" [tw] OR “Coronary Stenoses" [tw] OR "Coronary Thrombosis" [tw] OR "Coronary Thromboses" [tw] OR "Coronary Vasospasm" [tw] OR "Coronary Vasospasms" [tw] OR "Coronary-Subclavian Steal Syndrome"[tw] OR "ischemic event" $[\mathrm{tw}]$ OR "ischemic events" [tw] OR "ischaemic event" $[\mathrm{tw}]$ OR "ischaemic events" $[\mathrm{tw}]$ OR "Myocardial Ischemia"[Mesh] OR "Acute Coronary Syndrome"[tw] OR "Angina Pectoris" $[\mathrm{tw}]$ OR "Cardiogenic Shock" $[\mathrm{tw}]$ OR "Kounis Syndrome" $[\mathrm{tw}]$ OR "Microvascular Angina" [tw] OR "Myocardial Infarct" [tw] OR "Myocardial Infarction" [tw] OR "Myocardial Infarctions" [tw] OR "Myocardial Infarcts"[tw] OR "Myocardial Ischaemia" [tw] OR "Myocardial Ischemia" [tw] OR “Myocardial Reperfusion Injuries"[tw] OR “Myocardial Reperfusion Injury" $[\mathrm{tw}]$ OR "Stable Angina" $[\mathrm{tw}] \mathrm{OR}$ "Unstable Angina" [tw] OR "ischemic disease" $[\mathrm{tw}]$ OR "ischemic diseases" [tw] OR "ischaemic disease" [tw] OR "ischaemic diseases" $[\mathrm{tw}] \mathrm{OR}$ "coronary surgery" $[\mathrm{tw}] \mathrm{OR}$ "coronary artery surgery" [tw] OR "Coronary Artery Bypass"[Mesh] OR "Coronary Artery Bypass" [tw] OR "Coronary Vessels/surgery"[Mesh] OR "Catheterization"[Mesh] OR "Catheterization"[tw] OR "Catheterisation"[tw] OR Catheter*[tw] OR "Death, Sudden, Cardiac"[Mesh] OR "sudden cardiac death" [tw] OR "Cardiac Sudden Death" [tw] OR “Sudden Cardiac Arrest" $[\mathrm{tw}]$ OR “Karoshi Death" [tw] OR "coronary artery anatomy" $[\mathrm{tw}]$ OR "coronary artery pattern" $[\mathrm{tw}] \mathrm{OR}$ "coronary artery patterning" $[\mathrm{tw}] \mathrm{OR}$ "coronary artery patterns" [tw] OR "coronary outcome" [tw] OR "coronary outcomes" [tw] OR "Chest Pain"[Mesh] OR "thoracic pain*"[tw] OR "thorax pain" “Neuralgia”[Mesh] OR "nerve pain" ${ }^{*}[\mathrm{tw}]$ OR "neuronal pain*”[tw] OR “neuralg*”[tw] OR “atypical pain*" $[\mathrm{tw}] \mathrm{OR}$ “Denervation" [Mesh] OR “Denervat*" $[\mathrm{tw}] \mathrm{OR}$ "reinnervation" $[\mathrm{tw}] \mathrm{OR}$ "reinnervat ${ }^{* \prime}$ [tw] OR "re innervat*"[tw] OR “innervation" [Subheading] OR "innervation" [tw] OR “innervat" ${ }^{*}$ [tw] OR "coronary anatom" ${ }^{* \prime}[\mathrm{tw}]$ OR "Coronary Vessels/anatomy and histology" [mesh] OR "myocardial perfusion" [tw] OR "Myocardial Perfusion Imaging"[Mesh]) AND (english[la] OR german[la]) AND (“1994/01/01"[PDAT]: “3000/12/31”[PDAT])) OR ((“Arterial Switch Operation”"[majr] OR "Arterial Switch Operation"[ti] OR "Arterial Switch Operations" [ti] OR "arterial switch procedure"[ti] OR "arterial switch procedures"[ti] OR "arterial switch repair"[ti] OR "arterial switch surgery"[ti] OR "arterial switch"[ti] OR "Arterial Switch Technique"[ti] OR "Double Switch Operation"[ti] OR “Double Switch Operations"[ti] OR “Double Switch Procedure" [ti] OR “Double Switch Procedure ${ }^{* \prime}[\mathrm{ti}]$ OR "Double Switch Technique"[ti] OR "Double Switch"[ti] OR "Jatene Operation"[ti] OR “Jatene Procedure"[ti] OR "Jatene Technique"[ti]) AND ("outcomes"[tiab] OR "outcome"[tiab] OR "long term"[tiab] OR “longterm"[tiab] OR "experience"[tiab]) AND (english[la] OR german[la]) AND ("1994/01/01"[PDAT]: “3000/12/31"[PDAT]))) NOT ("ablation"[tw] OR "prenatal”[tw] OR "neonates" [ti] OR "neonate"[ti] OR "newborn"[ti] OR "newborns"[ti])

Embase: (( (*"Arterial Switch Operation"/OR “Arterial Switch Operation".ti,ab OR “Arterial Switch Operations".ti,ab OR "arterial switch procedure".ti,ab OR "arterial switch procedures".ti,ab OR "arterial switch repair".ti,ab OR "arterial switch surgery".ti,ab OR "arterial switch".ti,ab OR "Arterial Switch Technique".ti,ab OR "Double Switch Operation".ti,ab OR “Double Switch Operations".ti,ab OR “Double Switch Procedure".ti,ab OR "Double Switch Procedures".ti,ab OR “Double Switch Technique".ti,ab OR “Double Switch".ti,ab 
OR “Jatene Operation”.ti,ab OR “Jatene Procedure”.ti,ab OR “Jatene Technique”.ti,ab) AND ("coronary complications".ti,ab OR "coronary complication".ti,ab OR exp *"Coronary Artery Disease" /OR “Coronary Aneurysm".ti,ab OR “Coronary Aneurysms".ti,ab OR "Coronary Artery Disease".ti,ab OR "Coronary Artery Diseases".ti,ab OR “Coronary Disease".ti,ab OR “Coronary Diseases".ti,ab OR “Coronary Occlusion”.ti,ab OR “Coronary Occlusions".ti,ab OR “Coronary Restenosis".ti,ab OR “Coronary Restenoses".ti,ab OR “Coronary Stenosis".ti,ab OR "Coronary Stenoses".ti,ab OR “Coronary Thrombosis”.ti,ab OR “Coronary Thromboses”.ti,ab OR “Coronary Vasospasm”.ti,ab OR “Coronary Vasospasms”.ti,ab OR “Coronary-Subclavian Steal Syndrome".ti,ab OR “ischemic event".ti,ab OR “ischemic events".ti,ab OR "ischaemic event".ti,ab OR “ischaemic events".ti,ab OR exp *"Ischemic Heart Disease" /OR “Acute Coronary Syndrome”.ti,ab OR “Angina Pectoris".ti,ab OR “Cardiogenic Shock”.ti,ab OR “Kounis Syndrome”.ti,ab OR “Microvascular Angina".ti,ab OR "Myocardial Infarct”.ti,ab OR “Myocardial Infarction".ti,ab OR "Myocardial Infarctions".ti,ab OR "Myocardial Infarcts".ti,ab OR "Myocardial Ischaemia”.ti,ab OR “Myocardial Ischemia”.ti,ab OR “Myocardial Reperfusion Injuries".ti,ab OR “Myocardial Reperfusion Injury”.ti,ab OR “Stable Angina”.ti,ab OR “Unstable Angina".ti,ab OR “ischemic disease".ti,ab OR “ischemic diseases".ti,ab OR "ischaemic disease".ti,ab OR "ischaemic diseases".ti,ab OR "coronary surgery".ti,ab OR "coronary artery surgery".ti,ab OR *"Coronary Artery Bypass Graft" /OR "Coronary Artery Bypass".ti,ab OR exp *"coronary artery surgery" /OR exp *"Catheterization" /OR “Catheterization".ti,ab OR "Catheterisation".ti,ab OR Catheter*.ti,ab OR *"Sudden Cardiac Death" /OR "sudden cardiac death".ti,ab OR “Cardiac Sudden Death".ti,ab OR "Sudden Cardiac Arrest”.ti,ab OR “Karoshi Death".ti,ab OR "coronary artery anatomy".ti,ab OR "coronary artery pattern".ti,ab OR “coronary artery patterning".ti,ab OR “coronary artery patterns".ti,ab OR “coronary outcome”.ti,ab OR "coronary outcomes".ti,ab OR *'Thorax Pain"/OR "thoracic pain*".ti,ab OR "thorax pain*".ti,ab OR "chest pain*".ti,ab OR exp *"Neuralgia" /OR "nerve pain*".ti,ab OR "neuronal pain*".ti,ab OR "neuralg*".ti,ab OR "atypical pain*".ti,ab OR exp *"Denervation" /OR "Denervat" ${ }^{*}$.ti,ab OR "reinnervation".ti,ab OR "reinnervat*".ti,ab OR "re innervat".ti,ab OR exp "innervation" /OR “innervation".ti,ab OR “innervat".ti,ab OR "coronary anatom*".ti,ab OR "myocardial perfusion".ti,ab OR *"heart muscle perfusion" /OR *"Myocardial Perfusion Imaging" /) AND (english.la OR german.la)) OR ((*"Arterial Switch Operation" /OR "Arterial Switch Operation".ti OR "Arterial Switch Operations".ti OR “arterial switch procedure".ti OR “arterial switch procedures".ti OR “arterial switch repair".ti OR “arterial switch surgery”.ti OR "arterial switch".ti OR “Arterial Switch Technique”.ti OR “Double Switch Operation".ti OR “Double Switch Operations".ti OR “Double Switch Procedure”.ti OR “Double Switch Procedures".ti OR “Double Switch Technique".ti OR “Double Switch".ti OR “Jatene Operation".ti OR "Jatene Procedure".ti OR "Jatene Technique".ti) AND ("outcomes".ti,ab OR “outcome".ti,ab OR "long term".ti,ab OR “longterm”.ti,ab OR "experience”.ti,ab) AND (english.la OR german.la))) NOT ("ablation".ti,ab OR "prenatal".ti,ab OR “neonates".ti OR “neonate".ti OR "newborn".ti OR "newborns".ti)

Web of Science: ((ti=("Arterial Switch Operation" OR “Arterial Switch Operation" OR "Arterial Switch Operations" OR "arterial switch procedure" OR "arterial switch procedures" OR "arterial switch repair" OR "arterial switch surgery" OR "arterial switch" OR "Arterial Switch Technique" OR "Double Switch Operation" OR “Double Switch Operations" OR "Double Switch Procedure" OR "Double Switch Procedures" OR "Double Switch Technique" OR "Double Switch" OR “Jatene Operation" OR "Jatene Procedure" OR "Jatene Technique") AND ts=("coronary complications" OR "coronary complication" OR "Coronary Artery Disease" OR "Coronary Aneurysm" OR "Coronary Aneurysms" OR "Coronary Artery Disease" OR "Coronary Artery Diseases" OR "Coronary Disease" OR "Coronary Diseases" OR "Coronary Occlusion" OR "Coronary Occlusions" OR "Coronary Restenosis" OR "Coronary Restenoses" OR "Coronary Stenosis" OR “Coronary Stenoses" OR "Coronary Thrombosis" OR "Coronary Thromboses" OR "Coronary Vasospasm" OR "Coronary Vasospasms" OR "Coronary-Subclavian Steal Syndrome" OR "ischemic event" OR "ischemic events" OR "ischaemic event" OR "ischaemic events" OR "Ischemic Heart Disease" OR "Acute Coronary Syndrome" OR "Angina Pectoris" OR "Cardiogenic Shock" 
OR "Kounis Syndrome" OR "Microvascular Angina" OR “Myocardial Infarct" OR "Myocardial Infarction" OR "Myocardial Infarctions" OR “Myocardial Infarcts" OR “Myocardial Ischaemia" OR "Myocardial Ischemia" OR "Myocardial Reperfusion Injuries" OR "Myocardial Reperfusion Injury" OR "Stable Angina" OR "Unstable Angina" OR "ischemic disease" OR "ischemic diseases" OR "ischaemic disease" OR "ischaemic diseases" OR "coronary surgery" OR "coronary artery surgery" OR *"Coronary Artery Bypass Graft" OR "Coronary Artery Bypass" OR "coronary artery surgery" OR "Catheterization" OR "Catheterization" OR "Catheterisation" OR Catheter* OR *"Sudden Cardiac Death" OR "sudden cardiac death" OR "Cardiac Sudden Death" OR "Sudden Cardiac Arrest" OR "Karoshi Death" OR "coronary artery anatomy" OR "coronary artery pattern" OR "coronary artery patterning" OR "coronary artery patterns" OR "coronary outcome" OR "coronary outcomes" OR *"Thorax Pain" OR "thoracic pain*" OR "thorax pain*" OR "chest pain*" OR "Neuralgia" OR "nerve pain*" OR "neuronal pain*" OR "neuralg*" OR “atypical pain*" OR "Denervation" OR "Denervat" ${ }^{* \prime}$ OR "reinnervation" OR "reinnervat ${ }^{* \prime}$ OR "re innervat" ${ }^{*}$ " "innervation" OR "innervation" OR "innervat*" OR "coronary anatom*" OR "myocardial perfusion" OR *"heart muscle perfusion" OR "Myocardial Perfusion Imaging") AND la=(english OR german)) OR (ts=("Arterial Switch Operation" OR "Arterial Switch Operation" OR "Arterial Switch Operations" OR "arterial switch procedure" OR "arterial switch procedures" OR "arterial switch repair" OR "arterial switch surgery" OR "arterial switch" OR "Arterial Switch Technique" OR "Double Switch Operation" OR "Double Switch Operations" OR "Double Switch Procedure" OR "Double Switch Procedures" OR “Double Switch Technique" OR "Double Switch" OR "Jatene Operation" OR "Jatene Procedure" OR "Jatene Technique") AND ti=("coronary complications" OR "coronary complication" OR "Coronary Artery Disease" OR "Coronary Aneurysm" OR "Coronary Aneurysms" OR "Coronary Artery Disease" OR "Coronary Artery Diseases" OR "Coronary Disease" OR "Coronary Diseases" OR "Coronary Occlusion" OR "Coronary Occlusions" OR "Coronary Restenosis" OR "Coronary Restenoses" OR "Coronary Stenosis" OR "Coronary Stenoses" OR "Coronary Thrombosis" OR "Coronary Thromboses" OR "Coronary Vasospasm" OR "Coronary Vasospasms" OR "Coronary-Subclavian Steal Syndrome" OR "ischemic event" OR "ischemic events" OR "ischaemic event" OR "ischaemic events" OR "Ischemic Heart Disease" OR "Acute Coronary Syndrome" OR "Angina Pectoris" OR "Cardiogenic Shock" OR "Kounis Syndrome" OR “Microvascular Angina" OR "Myocardial Infarct" OR "Myocardial Infarction" OR "Myocardial Infarctions" OR "Myocardial Infarcts" OR "Myocardial Ischaemia" OR "Myocardial Ischemia" OR "Myocardial Reperfusion Injuries" OR "Myocardial Reperfusion Injury" OR "Stable Angina" OR "Unstable Angina" OR "ischemic disease" OR "ischemic diseases" OR "ischaemic disease" OR "ischaemic diseases" OR "coronary surgery" OR "coronary artery surgery" OR *"Coronary Artery Bypass Graft" OR "Coronary Artery Bypass" OR "coronary artery surgery" OR "Catheterization" OR "Catheterization" OR "Catheterisation" OR Catheter* OR *"Sudden Cardiac Death" OR "sudden cardiac death" OR "Cardiac Sudden Death" OR "Sudden Cardiac Arrest" OR "Karoshi Death" OR "coronary artery anatomy" OR "coronary artery pattern" OR "coronary artery patterning" OR "coronary artery patterns" OR "coronary outcome" OR "coronary outcomes" OR *"Thorax Pain" OR "thoracic pain" OR "thorax pain*" OR "chest pain*" OR "Neuralgia" OR "nerve pain*" OR "neuronal pain*" OR "neuralg*" OR "atypical pain" OR “Denervation" OR "Denervat" OR "reinnervation" OR "reinnervat" ${ }^{*}$ " OR "re innervat" OR "innervation" OR "innervation" OR "innervat*" OR "coronary anatom*" OR "myocardial perfusion" OR *"heart muscle perfusion" OR "Myocardial Perfusion Imaging")) OR (ti=(*"Arterial Switch Operation" OR "Arterial Switch Operation" OR "Arterial Switch Operations" OR "arterial switch procedure" OR "arterial switch procedures" OR "arterial switch repair" OR "arterial switch surgery" OR "arterial switch" OR "Arterial Switch Technique" OR "Double Switch Operation" OR "Double Switch Operations" OR "Double Switch Procedure" OR "Double Switch Procedures" OR "Double Switch Technique" OR "Double Switch" OR "Jatene Operation" OR "Jatene Procedure" OR "Jatene Technique") AND ts=("outcomes" OR "outcome" OR "long term" OR "longterm" OR "experience"))) NOT (ts=("ablation" 
OR "prenatal") OR ti=("neonates" OR “neonate" OR "newborn" OR “newborns")) AND la $=($ english OR german)

Cochrane Library: (("Arterial Switch Operation" OR "Arterial Switch Operation" OR "Arterial Switch Operations" OR "arterial switch procedure" OR "arterial switch procedures" OR "arterial switch repair" OR "arterial switch surgery" OR "arterial switch" OR "Arterial Switch Technique" OR "Double Switch Operation" OR "Double Switch Operations" OR "Double Switch Procedure" OR "Double Switch Procedures" OR "Double Switch Technique" OR "Double Switch" OR "Jatene Operation" OR "Jatene Procedure" OR "Jatene Technique"):ti, ab,kw AND ("coronary complications" OR "coronary complication" OR "Coronary Disease" OR "Coronary Aneurysm" OR "Coronary Aneurysms" OR "Coronary Artery Disease" OR "Coronary Artery Diseases" OR "Coronary Disease" OR "Coronary Diseases" OR "Coronary Occlusion" OR "Coronary Occlusions" OR "Coronary Restenosis" OR "Coronary Restenoses" OR "Coronary Stenosis" OR "Coronary Stenoses" OR "Coronary Thrombosis" OR “Coronary Thromboses" OR "Coronary Vasospasm" OR "Coronary Vasospasms" OR "Coronary-Subclavian Steal Syndrome" OR "ischemic event" OR "ischemic events" OR "ischaemic event" OR "ischaemic events" OR "Myocardial Ischemia" OR "Acute Coronary Syndrome" OR "Angina Pectoris" OR "Cardiogenic Shock" OR "Kounis Syndrome" OR "Microvascular Angina" OR "Myocardial Infarct" OR "Myocardial Infarction" OR "Myocardial Infarctions" OR "Myocardial Infarcts" OR "Myocardial Ischaemia" OR "Myocardial Ischemia" OR "Myocardial Reperfusion Injuries" OR "Myocardial Reperfusion Injury" OR "Stable Angina" OR "Unstable Angina" OR "ischemic disease" OR "ischemic diseases" OR "ischaemic disease" OR "ischaemic diseases" OR "coronary surgery" OR "coronary artery surgery" OR "Coronary Artery Bypass" OR "Coronary Artery Bypass" OR "Catheterization" OR "Catheterization" OR "Catheterisation" OR Catheter* OR "Death, Sudden, Cardiac" OR "sudden cardiac death" OR "Cardiac Sudden Death" OR "Sudden Cardiac Arrest" OR "Karoshi Death" OR "coronary artery anatomy" OR "coronary artery pattern" OR "coronary artery patterning" OR "coronary artery patterns" OR "coronary outcome" OR "coronary outcomes" OR "Chest Pain" OR "thoracic pain*" OR "thorax pain*" OR "chest pain*" OR "Neuralgia" OR "nerve pain*" OR "neuronal pain*" OR "neuralg*" OR "atypical pain*" OR "Denervation" OR "Denervat*" OR "reinnervation" OR "reinnervat" OR "re innervat" OR "innervation" OR "innervation" OR "innervat" OR "coronary anatom*" OR "coronary anatom*" OR "myocardial perfusion" OR *"heart muscle perfusion" OR "Myocardial Perfusion Imaging"):ti,ab,kw OR (ti=(*"Arterial Switch Operation" OR "Arterial Switch Operation" OR "Arterial Switch Operations" OR "arterial switch procedure" OR "arterial switch procedures" OR "arterial switch repair" OR "arterial switch surgery" OR "arterial switch" OR "Arterial Switch Technique" OR "Double Switch Operation" OR "Double Switch Operations" OR "Double Switch Procedure" OR "Double Switch Procedures" OR "Double Switch Technique" OR "Double Switch" OR "Jatene Operation" OR "Jatene Procedure" OR "Jatene Technique"):ti AND ts=("outcomes" OR "outcome" OR "long term" OR "longterm" OR “experience"):ti,ab,kw))

Emcare: see Embase.

\section{References}

1. Centers for Disease Control and Prevention (CDC). Improved national prevalence estimates for 18 selected major birth defectsUnited States, 1999-2001. MMWR Morb. Mortal. Wkly. Rep. 2006, 54, 1301-1305.

2. Martins, P.; Castela, E. Transposition of the great arteries. Orphanet J. Rare Dis. 2008, 3, 27. [CrossRef] [PubMed]

3. Jatene, A.D.; Fontes, V.F.; Paulista, P.P.; Souza, L.C.; Neger, F.; Galantier, M.; Sousa, J.E. Anatomic correction of transposition of the great vessels. J. Thorac. Cardiovasc. Surg. 1976, 72, 364-370. [CrossRef]

4. Jatene, A.D.; Fontes, V.F.; Paulista, P.P.; de Souza, L.C.; Neger, F.; Galantier, M.; Souza, J.E. Successful anatomic correction of transposition of the great vessels. A preliminary report. Arq. Bras. Cardiol. 1975, 28, 461-464. [PubMed]

5. Prêtre, R.; Tamisier, D.; Bonhoeffer, P.; Mauriat, P.; Pouard, P.; Sidi, D.; Vouhé, P. Results of the arterial switch operation in neonates with transposed great arteries. Lancet 2001,357, 1826-1830. [CrossRef]

6. Legendre, A.; Losay, J.; Touchot-Koné, A.; Serraf, A.; Belli, E.; Piot, J.D.; Lambert, V.; Capderou, A.; Planche, C. Coronary events after arterial switch operation for transposition of the great arteries. Circulation 2003, 108 (Suppl. 1), Ii186-Ii190. [CrossRef] 
7. Tanel, R.E.; Wernovsky, G.; Landzberg, M.J.; Perry, S.B.; Burke, R.P. Coronary artery abnormalities detected at cardiac catheterization following the arterial switch operation for transposition of the great arteries. Am. J. Cardiol. 1995, 76, 153-157. [CrossRef]

8. Bonnet, D.; Bonhoeffer, P.; Piéchaud, J.F.; Stümper, O.; Kachaner, J.; Sidi, D.; Vouhé, P.R. Coronary obstructions after reimplantation of the two coronary ostia in a single orifice during arterial switch operation for transposition of the great arteries. Eur. J. Cardiothorac. Surg. 1996, 10, 482. [CrossRef]

9. Veltman, C.E.; Beeres, S.; Kalkman, D.N.; Kelder, T.P.; Kiès, P.; Vliegen, H.W.; Hazekamp, M.G.; Delgado, V.; Kroft, L.J.M.; van der Wall, E.E.; et al. Variation in coronary anatomy in adult patients late after arterial switch operation: A computed tomography coronary angiography study. Ann. Thorac. Surg. 2013, 96, 1390-1397. [CrossRef]

10. Bonhoeffer, P.; Bonnet, D.; Piéchaud, J.F.; Stümper, O.; Aggoun, Y.; Villain, E.; Kachaner, J.; Sidi, D. Coronary artery obstruction after the arterial switch operation for transposition of the great arteries in newborns. J. Am. Coll. Cardiol. 1997, 29, 202-206. [CrossRef]

11. Stout, K.K.; Daniels, C.J.; Aboulhosn, J.A.; Bozkurt, B.; Broberg, C.S.; Colman, J.M.; Crumb, S.R.; Dearani, J.A.; Fuller, S.; Gurvitz, M.; et al. 2018 AHA/ACC Guideline for the Management of Adults with Congenital Heart Disease: A Report of the American College of Cardiology/American Heart Association Task Force on Clinical Practice Guidelines. J. Am. Coll. Cardiol. 2019, 73, e81-e192. [CrossRef] [PubMed]

12. Baumgartner, H.; De Backer, J.; Babu-Narayan, S.V.; Budts, W.; Chessa, M.; Diller, G.P.; Lung, B.; Kluin, J.; Lang, I.M.; Meijboom, F.; et al. 2020 ESC Guidelines for the management of adult congenital heart disease. Eur. Heart J. 2020, 42, 563-645. [CrossRef] [PubMed]

13. Stang, A. Critical evaluation of the Newcastle-Ottawa scale for the assessment of the quality of nonrandomized studies in meta-analyses. Eur. J. Epidemiol. 2010, 25, 603-605. [CrossRef] [PubMed]

14. Opolski, M.P.; Pregowski, J.; Kruk, M.; Witkowski, A.; Kwiecinska, S.; Lubienska, E.; Demkow, M.; Hryniewiecki, T.; Michalek, P.; Ruzyllo, W.; et al. Prevalence and characteristics of coronary anomalies originating from the opposite sinus of Valsalva in 8,522 patients referred for coronary computed tomography angiography. Am. J. Cardiol. 2013, 111, 1361-1367. [CrossRef] [PubMed]

15. Jegatheeswaran, A.; Devlin, P.J.; McCrindle, B.W.; Williams, W.G.; Jacobs, M.L.; Blackstone, E.H.; DeCampli, W.M.; Caldarone, C.A.; Gaynor, J.W.; Kirklin, J.K.; et al. Features associated with myocardial ischemia in anomalous aortic origin of a coronary artery: A Congenital Heart Surgeons' Society study. J. Thorac. Cardiovasc. Surg. 2019, 158, 822-834.e3. [CrossRef]

16. Fricke, T.A.; Bell, D.; Daley, M.; d'Udekem, Y.; Brizard, C.P.; Alphonso, N.; Konstantinov, I.E. The influence of coronary artery anatomy on mortality after the arterial switch operation. J. Thorac. Cardiovasc. Surg. 2020, 160, 191-199.e1. [CrossRef] [PubMed]

17. Khairy, P.; Clair, M.; Fernandes, S.M.; Blume, E.D.; Powell, A.J.; Newburger, J.W.; Landzberg, M.J.; Mayer, J.E., Jr. Cardiovascular outcomes after the arterial switch operation for D-transposition of the great arteries. Circulation 2013, 127, 331-339. [CrossRef]

18. Moll, M.; Moll, J.A.; Moll, J.J.; Łubisz, M.; Michalak, K.W. Intramural coronary pattern in patients with transposition: Incidence and impact on follow-up. Eur. J. Cardiothorac. Surg. 2020, 58, 145-152. [CrossRef] [PubMed]

19. Kempny, A.; Wustmann, K.; Borgia, F.; Dimopoulos, K.; Uebing, A.; Li, W.; Chen, S.S.; Piorkowski, A.; Radley-Smith, R.; Yacoub, M.H.; et al. Outcome in adult patients after arterial switch operation for transposition of the great arteries. Int. J. Cardiol. 2013, 167, 2588-2593. [CrossRef]

20. Tobler, D.; Williams, W.G.; Jegatheeswaran, A.; Van Arsdell, G.S.; McCrindle, B.W.; Greutmann, M.; Oechslin, E.N.; Silversides, C.K. Cardiac outcomes in young adult survivors of the arterial switch operation for transposition of the great arteries. J. Am. Coll. Cardiol. 2010, 56, 58-64. [CrossRef]

21. Santens, B.; Van De Bruaene, A.; De Meester, P.; Gewillig, M.; Troost, E.; Claus, P.; Bogaert, J.; Budts, W. Outcome of arterial switch operation for transposition of the great arteries. A 35-year follow-up study. Int. J. Cardiol. 2020, 316, 94-100. [CrossRef] [PubMed]

22. Baruteau, A.E.; Vergnat, M.; Kalfa, D.; Delpey, J.G.; Ly, M.; Capderou, A.; Lambert, V.; Belli, E. Long-term outcomes of the arterial switch operation for transposition of the great arteries and ventricular septal defect and/or aortic arch obstruction. Interact. Cardiovasc. Thorac. Surg. 2016, 23, 240-246. [CrossRef] [PubMed]

23. Lo Rito, M.; Fittipaldi, M.; Haththotuwa, R.; Jones, T.J.; Khan, N.; Clift, P.; Brawn, W.J.; Barron, D.J. Long-term fate of the aortic valve after an arterial switch operation. J. Thorac. Cardiovasc. Surg. 2015, 149, 1089-1094. [CrossRef] [PubMed]

24. Vida, V.L.; Zanotto, L.; Zanotto, L.; Triglia, L.T.; Bellanti, E.; Castaldi, B.; Padalino, M.A.; Gasperetti, A.; Battista, F.; Varnier, M.; et al. Arterial switch operation for transposition of the great arteries: A single-centre 32-year experience. J. Card. Surg. 2019, 34, 1154-1161. [CrossRef]

25. Oda, S.; Nakano, T.; Sugiura, J.; Fusazaki, N.; Ishikawa, S.; Kado, H. Twenty-eight years' experience of arterial switch operation for transposition of the great arteries in a single institution. Eur. J. Cardiothorac. Surg. 2012, 42, 674-679. [CrossRef] [PubMed]

26. Raissadati, A.; Nieminen, H.; Sairanen, H.; Jokinen, E. Outcomes after the Mustard, Senning and arterial switch operation for treatment of transposition of the great arteries in Finland: A nationwide 4-decade perspective. Eur. J. Cardiothorac. Surg. 2017, 52, 573-580. [CrossRef]

27. Ruys, T.P.; van der Bosch, A.E.; Cuypers, J.A.; Witsenburg, M.; Helbing, W.A.; Bogers, A.J.; van Domburg, R.; McGhie, J.S.; Geleijnse, M.L.; Henrichs, J.; et al. Long-term outcome and quality of life after arterial switch operation: A prospective study with a historical comparison. Congenit. Heart Dis. 2013, 8, 203-210. [CrossRef]

28. Hörer, J.; Schreiber, C.; Cleuziou, J.; Vogt, M.; Prodan, Z.; Busch, R.; Holper, K.; Lange, R. Improvement in long-term survival after hospital discharge but not in freedom from reoperation after the change from atrial to arterial switch for transposition of the great arteries. J. Thorac. Cardiovasc. Surg. 2009, 137, 347-354. [CrossRef] 
29. Shivaram, P.; Padiyath, A.; Bai, S.; Gossett, J.M.; Collins, R.T. Utility of Follow-Up Annual Echocardiograms in Patients With Complete Transposition of the Great Arteries After Arterial Switch Operations. Am. J. Cardiol. 2018, 122, 1972-1976. [CrossRef]

30. Lim, H.G.; Kim, W.H.; Lee, J.R.; Kim, Y.J. Long-term results of the arterial switch operation for ventriculo-arterial discordance. Eur. J. Cardiothorac. Surg. 2013, 43, 325-334. [CrossRef]

31. Choi, B.S.; Kwon, B.S.; Kim, G.B.; Bae, E.J.; Noh, C.I.; Choi, J.Y.; Yun, Y.S.; Kim, W.H.; Lee, J.R.; Kim, Y.J. Long-term outcomes after an arterial switch operation for simple complete transposition of the great arteries. Korean Circ. J. 2010, 40, 23-30. [CrossRef]

32. Rudra, H.S.; Mavroudis, C.; Backer, C.L.; Kaushal, S.; Russell, H.; Stewart, R.D.; Webb, C.; Sullivan, C. The arterial switch operation: 25-year experience with 258 patients. Ann. Thorac. Surg. 2011, 92, 1742-1746. [CrossRef]

33. Gerelli, S.; Pontailler, M.; Rochas, B.; Angeli, E.; Van Steenberghe, M.; Bonnet, D.; Vouhé, P.; Raisky, O. Single coronary artery and neonatal arterial switch operation: Early and long-term outcomes. Eur. J. Cardiothorac. Surg. 2017, 52, 90-95. [CrossRef] [PubMed]

34. De Praetere, H.; Vandesande, J.; Rega, F.; Daenen, W.; Marc, G.; Eyskens, B.; Heying, R.; Cools, B.; Meyns, B. 20 years of arterial switch operation for simple TGA. Acta Chir. Belg. 2014, 114, 92-98. [CrossRef]

35. Hayes, D.A.; Jones, S.; Quaegebeur, J.M.; Richmond, M.E.; Andrews, H.F.; Glickstein, J.S.; Chen, J.M.; Bacha, E.; Liberman, L. Primary arterial switch operation as a strategy for total correction of Taussig-Bing anomaly: A 21-year experience. Circulation 2013, 128, S194-S198. [CrossRef] [PubMed]

36. Hutter, P.A.; Kreb, D.L.; Mantel, S.F.; Hitchcock, J.F.; Meijboom, E.J.; Bennink, G.B. Twenty-five years' experience with the arterial switch operation. J. Thorac. Cardiovasc. Surg. 2002, 124, 790-797. [CrossRef] [PubMed]

37. Manso, P.H.; Amaral, F.T.; Júnior, T.J.; Jurca, M.C.; Haddad, J.; Vicente, W.V.; Sgarbieri, R.N.; Carmona, F. Outcomes of Patients After Arterial Switch Operation: 18 Years of Experience in a Single Medium-Volume Center. Pediatr. Cardiol. 2015, 36, 1657-1661. [CrossRef] [PubMed]

38. El-Segaier, M.; Lundin, A.; Hochbergs, P.; Jögi, P.; Pesonen, E. Late coronary complications after arterial switch operation and their treatment. Catheter. Cardiovasc. Interv. 2010, 76, 1027-1032. [CrossRef] [PubMed]

39. Görler, H.; Ono, M.; Thies, A.; Lunkewitz, E.; Westhoff-Bleck, M.; Haverich, A.; Breymann, T.; Boethig, D. Long-term morbidity and quality of life after surgical repair of transposition of the great arteries: Atrial versus arterial switch operation. Interact. Cardiovasc. Thorac. Surg. 2011, 12, 569-574. [CrossRef]

40. Arnaz, A.; Sarioglu, T.; Yalcinbas, Y.; Erek, E.; Turkoz, R.; Oktay, A.; Saygili, A.; Altun, D.; Sarioglu, A. Coronary artery bypass grafting in children. J. Card. Surg. 2018, 33, 29-34. [CrossRef]

41. Shim, M.S.; Jun, T.G.; Yang, J.H.; Park, P.W.; Kang, I.S.; Huh, J.; Song, J.Y. Current expectations of the arterial switch operation in a small volume center: A 20-year, single-center experience. J. Cardiothorac. Surg. 2016, 11, 34. [CrossRef]

42. Michalak, K.W.; Sobczak-Budlewska, K.; Moll, J.J.; Szymczyk, K.; Moll, J.A.; Niwald, M.; Dryżek, P.; Moll, M. Can we predict potentially dangerous coronary patterns in patients with transposition of the great arteries after an arterial switch operation? Cardiol. Young 2019, 29, 1350-1355. [CrossRef] [PubMed]

43. Tobler, D.; Motwani, M.; Wald, R.M.; Roche, S.L.; Verocai, F.; Iwanochko, R.M.; Greenwood, J.P.; Oechslin, E.N.; Crean, A.M. Evaluation of a comprehensive cardiovascular magnetic resonance protocol in young adults late after the arterial switch operation for d-transposition of the great arteries. J. Cardiovasc. Magn. Reson. 2014, 16, 98. [CrossRef]

44. Lalezari, S.; Bruggemans, E.F.; Blom, N.A.; Hazekamp, M.G. Thirty-year experience with the arterial switch operation. Ann. Thorac. Surg. 2011, 92, 973-979. [CrossRef] [PubMed]

45. Hayes, A.M.; Baker, E.J.; Kakadeker, A.; Parsons, J.M.; Martin, R.P.; Radley-Smith, R.; Qureshi, S.A.; Yacoub, M.; Maisey, M.N.; Tynan, M. Influence of anatomic correction for transposition of the great arteries on myocardial perfusion: Radionuclide imaging with technetium-99m 2-methoxy isobutyl isonitrile. J. Am. Coll. Cardiol. 1994, 24, 769-777. [CrossRef]

46. Quarrie, R.; Kopf, G.S.; Hashim, S. Left main coronary artery occlusion in an asymptomatic patient: Late complication after arterial switch operation. J. Card. Surg. 2016, 31, 599-600. [CrossRef] [PubMed]

47. Stoll, V.M.; Hudsmith, L.E.; Drury, N.E.; Barron, D.J. Late complication of intramural coronary transfer during the arterial switch operation. Interact. Cardiovasc. Thorac. Surg. 2019, 28, 638-639. [CrossRef]

48. Ueki, Y.; Kadner, A.; Gräni, C.; Räber, L. ST-Segment Elevation Myocardial Infarction Due to Optical Coherence TomographyDetected Coronary Artery Compression Following Supravalvular Pulmonary Artery Patchplasty 18 Years after Switch Procedure. JACC Cardiovasc. Interv. 2018, 11, e149-e151. [CrossRef]

49. Hamada, S.; Frick, M.; Marx, N.; Brehmer, K.; Manka, R. Severe myocardial ischaemia after neonatal arterial switch operation. Eur. Heart J. 2015, 36, 3214. [CrossRef]

50. Stoll, V.M.; Drury, N.E.; Thorne, S.; Selman, T.; Clift, P.; Chong, H.; Thompson, P.J.; Morris, R.K.; Hudsmith, L.E. Pregnancy Outcomes in Women with Transposition of the Great Arteries after an Arterial Switch Operation. JAMA Cardiol. 2018 , 3, $1119-1122$. [CrossRef] [PubMed]

51. Tobler, D.; Fernandes, S.M.; Wald, R.M.; Landzberg, M.; Salehian, O.; Siu, S.C.; Colman, J.M.; Sermer, M.; Silversides, C.K. Pregnancy outcomes in women with transposition of the great arteries and arterial switch operation. Am. J. Cardiol. 2010, 106, 417-420. [CrossRef]

52. Fricke, T.A.; Konstantinov, I.E.; Grigg, L.E.; Zentner, D. Pregnancy Outcomes in Women after the Arterial Switch Operation. Heart Lung Circ. 2019, 29, 1087-1092. [CrossRef] 
53. Horiuchi, C.; Kamiya, C.A.; Ohuchi, H.; Miyoshi, T.; Tsuritani, M.; Iwanaga, N.; Neki, R.; Niwa, K.; Kurosaki, K.; Ichikawa, H.; et al. Pregnancy outcomes and mid-term prognosis in women after arterial switch operation for dextro-transposition of the great arteries-Tertiary hospital experiences and review of literature. J. Cardiol. 2019, 73, 247-254. [CrossRef] [PubMed]

54. Cetta, F.; Bonilla, J.J.; Lichtenberg, R.C.; Stasior, C.; Troman, J.E.; Deleon, S.Y. Anatomic correction of dextrotransposition of the great arteries in a 36-year-old patient. Mayo Clin. Proc. 1997, 72, 245-247. [CrossRef] [PubMed]

55. Maeda, T.; Sakamoto, T.; Nagashima, M.; Hiramatsu, T.; Yamazaki, K. Long-Term Outcome of Arterial Switch Operation Conversion After Failed Senning/Mustard Procedure. Ann. Thorac. Surg. 2016, 102, 1573-1579. [CrossRef] [PubMed]

56. Padalino, M.A.; Stellin, G.; Brawn, W.J.; Fasoli, G.; Daliento, L.; Milanesi, O.; Casarotto, D. Arterial switch operation after left ventricular retraining in the adult. Ann. Thorac. Surg. 2000, 70, 1753-1757. [CrossRef]

57. Watanabe, N.; Mainwaring, R.D.; Carrillo, S.A.; Lui, G.K.; Reddy, V.M.; Hanley, F.L. Left Ventricular Retraining and Late Arterial Switch for D-Transposition of the Great Arteries. Ann. Thorac. Surg. 2015, 99, 1655-1661. [CrossRef] [PubMed]

58. Uno, Y.; Morita, K.; Ko, Y.; Kinouchi, K. Double switch operation for congenitally corrected transposition of the great arteries after two-staged pulmonary artery banding. Jpn. J. Thorac. Cardiovasc. Surg. 2006, 54, 40-43. [CrossRef]

59. Konstantinov, I.E.; Fricke, T.A.; d'Udekem, Y.; Radford, D.J. Translocation of a single coronary artery from the nonfacing sinus in the arterial switch operation: Long-term patency of the interposition graft. J. Thorac. Cardiovasc. Surg. 2010, 140, 1193-1194. [CrossRef]

60. Possner, M.; Buechel, R.R.; Vontobel, J.; Mikulicic, F.; Gräni, C.; Benz, D.C.; Clerc, O.F.; Fuchs, T.A.; Tobler, D.; Stambach, D.; et al. Myocardial blood flow and cardiac sympathetic innervation in young adults late after arterial switch operation for transposition of the great arteries. Int. J. Cardiol. 2020, 299, 110-115. [CrossRef]

61. Kuehn, A.; Vogt, M.; Schwaiger, M.; Ewert, P.; Hauser, M. Ventricular sympathetic innervation in patients with transposition of the great arteries after arterial switch operation and Rastelli procedure: Impact of arterial dissection and coronary reimplantation. Circ. J. 2014, 78, 1717-1722. [CrossRef] [PubMed]

62. Vogel, M.; Smallhorn, J.F.; Gilday, D.; Benson, L.N.; Ash, J.; Williams, W.G.; Freedom, R.M. Assessment of myocardial perfusion in patients after the arterial switch operation. J. Nucl. Med. 1991, 32, 237-241. [PubMed]

63. Ou, P.; Celermajer, D.S.; Marini, D.; Agnoletti, G.; Vouhé, P.; Brunelle, F.; Le Quan Sang, K.H.; Thalabard, J.C.; Sidi, D.; Bonnet, D. Safety and accuracy of 64-slice computed tomography coronary angiography in children after the arterial switch operation for transposition of the great arteries. JACC Cardiovasc. Imaging 2008, 1, 331-339. [CrossRef] [PubMed]

64. Morfaw, F.; Leenus, A.; Mbuagbaw, L.; Anderson, L.N.; Dillenburg, R.; Thabane, L. Outcomes after corrective surgery for congenital dextro-transposition of the arteries using the arterial switch technique: A scoping systematic review. Syst. Rev. 2020, 9, 231. [CrossRef]

65. Koppel, C.J.; Driesen, B.W.; de Winter, R.J.; van den Bosch, A.E.; van Kimmenade, R.; Wagenaar, L.J.; Jukema, J.W.; Hazekamp, M.G.; van der Kley, F.; Jongbloed, M.R.M.; et al. The first multicentre study on coronary anomalies in the Netherlands: MuSCAT. Neth. Heart J. 2021, 29, 311-317. [CrossRef]

66. Van der Bom, T.; van der Palen, R.L.; Bouma, B.J.; van Veldhuisen, S.L.; Vliegen, H.W.; Konings, T.C.; Zwinderman, A.H.; Blom, N.A.; Koolbergen, D.R.; Hazekamp, M.G.; et al. Persistent neo-aortic growth during adulthood in patients after an arterial switch operation. Heart 2014, 100, 1360-1365. [CrossRef] 